\title{
Patientinnenzufriedenheit mit der Operations- aufklärung der Schweizerischen Gesellschaft für Gynäkologie und Geburtshilfe
}

Die vorliegende Studie wurde als Originalarbeit in der Märzausgabe 2006 von Mayo Clinic Proceedings publiziert [1] und wird hier in gekürzter deutscher Fassung wiedergegeben. Die Studie wurde durch die Schweizerische Gesellschaft für Gynäkologie und Geburtshilfe sowie durch die Gesundheitsförderung Schweiz finanziert.

Margrit Kessler ${ }^{a}$,

Amina T. Ghulam ${ }^{b}$,

Lucas M. Bachmann',

Urs Haller ${ }^{b}$,

Thomas M. Kessler ${ }^{a}$

a Schweizerische PatientenOrganisation (SPO), Zürich

b Departement für Gynäkologie und Geburtshilfe,

Universitätsspital Zürich, Zürich

c Horten Zentrum,

Universität Zürich, Zürich
Korrespondenz: Margrit Kessler

Schweizerische Patientenorganisation (SPO)

Häringstrasse 20

CH-8023 Zürich

Tel. 0717551657

Fax 0717552357

margrit.kessler@spo.ch

\section{Einleitung}

Verschiedene Faktoren wie Informationsbedürfnis, Respektierung der Autonomie des Patienten und nicht zuletzt medizin-rechtliche Überlegungen sind zu integrierenden Bestandteilen der Patientenaufklärung geworden. Eine verständliche präoperative Information soll es dem Patienten ermöglichen, bei der geplanten Behandlung mitzuentscheiden. Viele Aufklärungsprotokolle sind kompliziert und nur schwer verständlich [2-5]. Patienten sind häufig überfordert und können sich oft nicht an Einzelheiten erinnern [6, 7]. Mehrere Autoren empfehlen, die Patienten mündlich und schriftlich über einen Eingriff aufzuklären [8-13]. In der Schweiz wurde in den letzten Jahren viel zur Verbesserung und Weiterentwicklung der präoperativen Patienteninformation unternommen [12, 14-16]. Ziel der vorliegenden Arbeit war es, die Patientinnenzufriedenheit mit der Aufklärung über Eingriffe in der Gynäkologie und Geburtshilfe zu untersuchen.

\section{Patientinnen und Methoden}

Die Studie wurde in enger Zusammenarbeit der Schweizerischen Gesellschaft für Gynäkologie und Geburtshilfe (SGGG), des Rechtsdiensts der FMH, des Institutes für Sozial- und Präventivmedizin der Universität Zürich mit der Schweizerischen Patientenorganisation (SPO) ausgearbeitet. Unter Berücksichtigung der deutsch-, französisch- und italienisch-sprachigen Bevölkerungsanteile der Schweiz wurde proportional zu diesen Sprachregionen in 11 Schweizer Kliniken für Gynäkologie und Geburtshilfe die Patientinnenzufriedenheit mit der Operationsaufklärung evaluiert. Die Aufklä-

\section{Summary}

Patients' satisfaction with the preoperative informed consent procedure in obstetrics and gynecology was assessed by questionnaire in 11 Swiss hospitals. 3888 (56\%) of 6970 women treated between March 2001 and April 2002 received a questionnaire and were enrolled. Almost 90\% considered the written and oral information to be good or excellent and more than $80 \%$ did not desire further written information. In multivariate analysis, Turkish and Serbo-Croat language and poor rating of the written description of the planned operation were the only variables significantly associated with discontent. The combined written and oral preoperative information presented is well adapted to patients' informative wishes and needs; it allows for a structured conversation, facilitates documentation and offers valid legal proof that adequate information has been provided. Therefore, close collaboration between the national patient organization and the expert judiciary and medical societies of the corresponding country is strongly recommended to improve the informed consent procedure.

Keywords: patients' satisfaction, informed consent, questionnaire survey, obstetrics and gynecology 
rungsprotokolle können auf der Homepage der SGGG (www.sggg.ch) heruntergeladen werden. Bei Patientinnen mit Sprachschwierigkeiten wurde ein Dolmetscher beigezogen. Die Studie dauerte von März 2001 bis April 2002.

\section{Operationsaufklärung}

Am Tag des Spitaleintritts wurde ein standardisiertes, operationsspezifisches Aufklärungsprotokoll mit einer detaillierten Beschreibung der geplanten Operation und des Spitalaufenthaltes abgegeben. Dabei war genügend Platz für die Dokumentation des individuellen Aufklärungsgesprächs einschliesslich Operationsskizze und Hinweise auf andere Behandlungsmöglichkeiten vorhanden. Am Ende des Aufklärungsgesprächs wurde das Protokoll von

Tabelle 1

Patientinnencharakteristika.

\begin{tabular}{lrr} 
Alter der Patientinnen $(\mathbf{n}=\mathbf{3 7 7 7})$ & $\mathbf{n}$ & $\%$ \\
\hline $16-20$ & 109 & 3 \\
\hline $21-30$ & 560 & 15 \\
\hline $31-40$ & 1041 & 28 \\
\hline $41-50$ & 759 & 20 \\
\hline $51-60$ & 546 & 14 \\
\hline $61-70$ & 421 & 11 \\
\hline $71-80$ & 263 & 7 \\
\hline $81-90$ & 77 & 2 \\
$>90$ & 1 & 0
\end{tabular}

Wohnort $(\mathrm{n}=\mathbf{3 7 2 5})$

Wohnen in der Stadt (>10 000 Einwohner)

Sprache $(n=3732)$

Deutsch

2777

74

Italienisch

277

Französisch

330

Serbokroatisch

Türkisch

Andere

7

9

2

1

6

Operationsaufklärung durch $(\mathrm{n}=3647)$

Ärztin

Arzt

Durchführung des Eingriffs $(n=3734)$

Erkrankung $(\mathrm{n}=3661)$
Patientin und Arzt unterschrieben und eine Kopie davon der Patientin abgeben.

\section{Fragebogen}

Der Fragebogen war anonymisiert und bestand aus zwei Teilen, einem für den Arzt und einem für die Patientin. Beide Fragebogenteile waren mit der gleichen Nummer codiert, damit der Fragebogen später wieder zusammengefügt werden konnte.

\section{Statistische Analyse}

Zusätzlich zur deskriptiven statistischen Analyse wurde die Patientinnenzufriedenheit durch multivariate logistische Regressionsmodelle untersucht.

\section{Resultate}

Während der 14monatigen Studiendauer (März 2001-April 2002) wurden insgesamt 6970 Frauen behandelt. Davon erhielten 3888 Patientinnen (56\%) einen Fragebogen und wurden in die Studie aufgenommen (Tab. 1).

\section{Schriftliche Operationsaufklärung}

Die meisten Patientinnen beurteilten die schriftliche Aufklärung über die Notwendigkeit der Operation, die Operationstechnik, die Verständlichkeit der Operationsskizze, die Risiken und Komplikationen, die postoperative Behandlung sowie die Verständlichkeit der schriftlichen Operationsaufklärung als gut oder ausgezeichnet (Abb. 1). Über 80\% wünschten keine zusätzlichen schriftlichen Informationen.

95\% der Patientinnen waren mit dem Aufklärungsprotokoll zufrieden, 3\% fanden es zu wenig ausführlich und $2 \%$ wünschten weniger schriftliche Informationen.

Für $45 \%$ der Patientinnen sollte die schriftliche Operationsaufklärung gleichentags mit der Indikationsstellung für den Eingriff erfolgen. $16 \%$ wünschten zwei oder mehr Tage vor dem Eingriff und $24 \%$ einen Tag vor dem Eingriff aufgeklärt zu werden. Für 15\% war der Zeitpunkt der Operationsaufklärung nicht wichtig.

$51 \%$ der Patientinnen fühlten sich durch das Aufklärungsprotokoll beruhigt, 7\% wurden dadurch verängstigt und $42 \%$ wurden weder beruhigt noch verängstigt.

Nahezu alle (98\%) Patientinnen wurden bereits vor Spitaleintritt durch die zuweisenden Ärzte über den Grund der Operation informiert.

\section{Mündliche Operationsaufklärung}

Nur 38\% der Patientinnen gaben an, sie seien über Behandlungsalternativen informiert worden. 95\% glaubten, dass die vorgeschlagene 
Operation die einzige Behandlungsmöglichkeit sei. Die meisten Patientinnen beurteilten die Informationen über Behandlungsalternativen, die Notwendigkeit der Operation und die Verständlichkeit der mündlichen Operationsaufklärung als gut oder ausgezeichnet (Abb. 2).

Über 90\% der Patientinnen hatten ihren Operateur vor der Operation kennengelernt. Nahezu alle (99\%) Patientinnen stellten während des Informationsgesprächs Fragen. Für 45\% sollte die mündliche Operationsaufklärung gleichentags mit der Indikationsstellung für den Eingriff erfolgen. 12\% wünschten 2 oder mehr Tage vor dem Eingriff und 30\% einen Tag vor dem Eingriff aufgeklärt zu werden. Für 11\% war der Zeitpunkt der Operationsaufklärung nicht wichtig.

$61 \%$ der Patientinnen waren nach dem Gespräch mit dem Arzt beruhigt und nur 6\% verängstigt. $33 \%$ wurden durch das Aufklärungsgespräch nicht beeinflusst. Fast alle (97\%) Patientinnen wollten im Fall eines weiteren chirurgischen Eingriffs wieder auf dieselbe Art aufgeklärt werden.

\section{Abbildung 1}

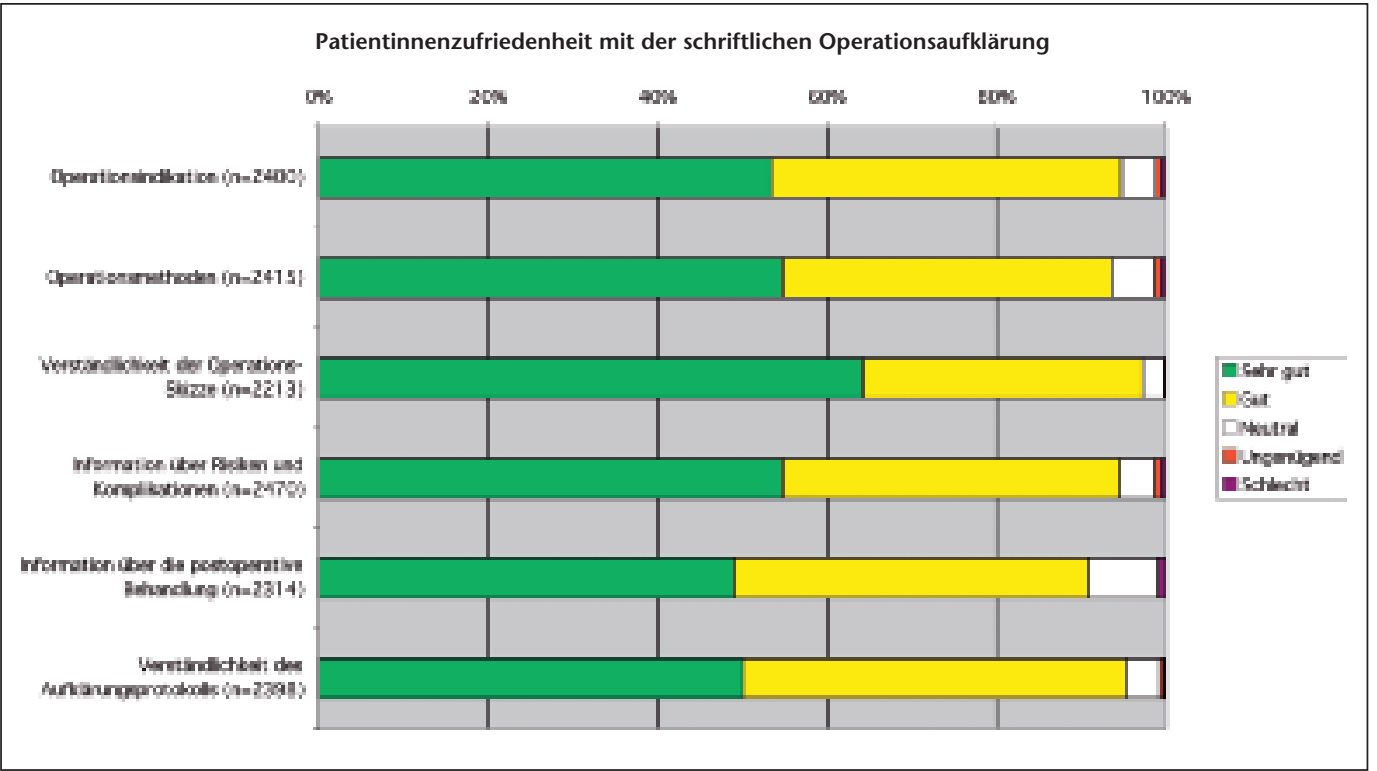

Abbildung 2

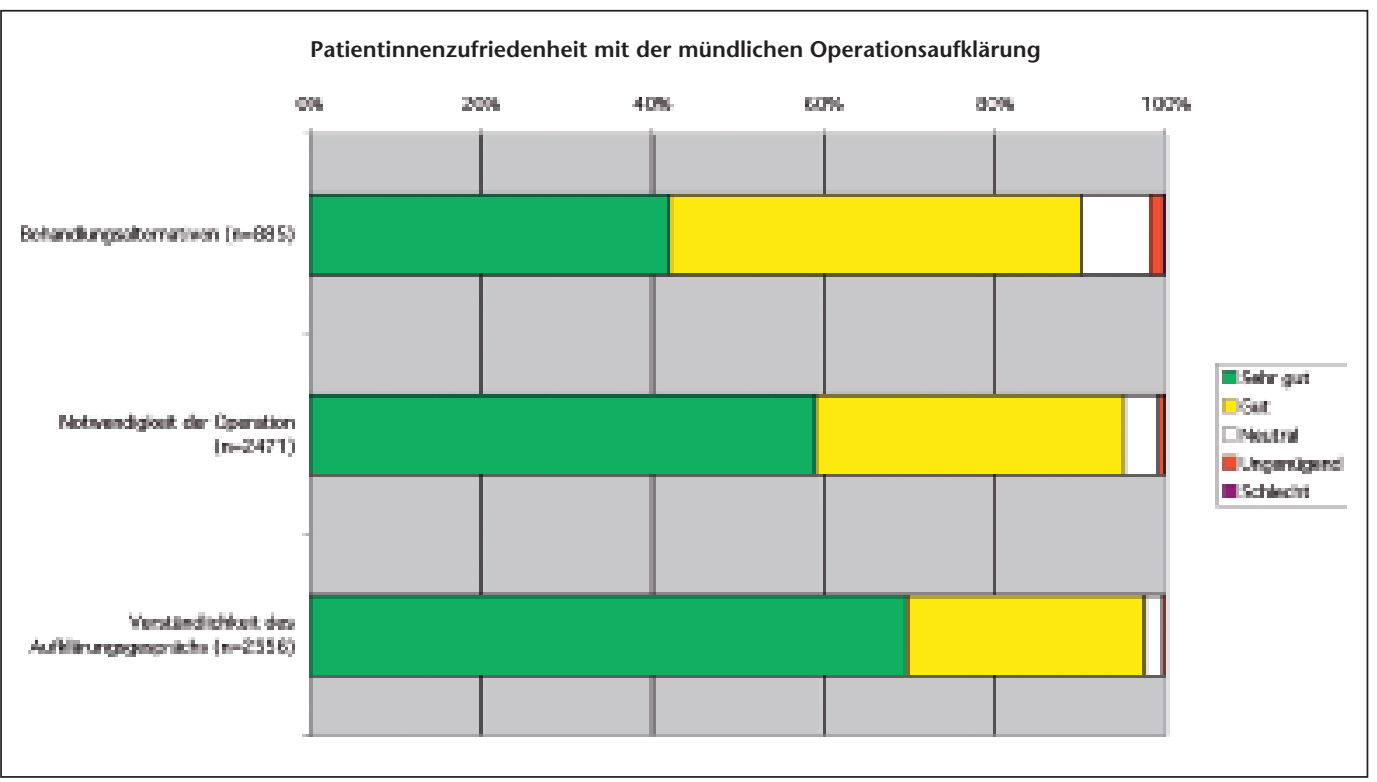




\section{Multivariate Analyse}

Insgesamt waren nur 2\% der Patientinnen unzufrieden mit der Operationsaufklärung. Türkische (odds ratio [OR] 6.7, 95\% Vertrauensintervall [CI 2.0-22.4, p = 0.002) und serbokroatische (OR 8.0, 95\% CI 2.4-27.4, p = 0.001) Muttersprache sowie eine schlechte schriftliche Beschreibung der geplanten Operation (OR 3.1, 95\% CI 1.1-9.0, p = 0.03) waren die einzigen mit Unzufriedenheit assoziierten Faktoren.

\section{Diskussion}

Um bei invasiven medizinischen Behandlungen und Abklärungen mitentscheiden zu können, ist eine angemessene Aufklärung des Patienten in allgemeinverständlicher Sprache unumgänglich. Die Aufklärung sollte Vorteile, Risiken und mögliche Komplikationen sowie alternative Behandlungsmöglichkeiten des geplanten Eingriffes aufzeigen [17]. Verschiedene Studien zeigten, dass schriftlich aufgeklärte Patienten den Eingriff besser verstehen [18] und sich auch nach der Operation besser an die Aufklärung $[19,20]$ erinnern. Die Patienten können die schriftliche Information mehrmals lesen, Angehörigen und Freunden vorlegen und mit ihnen den bevorstehenden Eingriff diskutieren. Wie wichtig dies für die Patienten ist, wird dadurch belegt, dass zwei Drittel der Patienten bei einer Studie über die Operationsaufklärung das Aufklärungsprotokoll vor dem Eingriff nochmals gelesen haben [21]. In Stresssituationen können viele Patienten mündliche Informationen nicht genügend aufnehmen [22,23]. Eine kombinierte schriftliche und mündliche Aufklärung ist patientenfreundlicher und deshalb zu favorisieren $[5,21]$. Das Aufklärungsgespräch kann zielgerichteter erfolgen und die Qualität wird deutlich verbessert, wenn der Patient die schriftliche Information bereits im voraus lesen konnte [24]. Das Aufklärungsprotokoll soll möglichst kurz und in laienverständlicher Sprache verfasst sein. Eine Operationsskizze als Zusatz zum Text erleichtert dem Patienten das Verständnis der Anatomie und der Operationstechnik [25].

Gewisse Autoren vertreten die Ansicht, dass die Aufklärung über Risiken und Komplikationen unnötige Ängste verursacht [26, 27]. Andere wiederum berichten, dass eine bessere Kenntnis der Behandlung die Angst verringert [6, 28-33]. In unserer Studie wurden mehr als die Hälfte der Patientinnen durch die Aufklärung beruhigt und nur 7\% verängstigt. Die Patienten wollen informiert werden und bei ihrer Behandlung mitentscheiden [34]. So möchten auch die meisten Krebspatienten so viel wie möglich über ihre Krankheit und Behandlung wissen [35].
Bei unseren Patientinnen war der bevorzugte Zeitpunkt für die Aufklärung der Tag, an dem die Entscheidung zur Operation getroffen wurde. Dies wurde auch in anderen Studien gefunden [11, 12, 14]. Der Patient sollte den Operateur bereits vor dem Spitaleintritt kennenlernen [12, 36], um ein Vertrauensverhältnis zum Operateur aufbauen zu können [15].

Nur wenige Patientinnen gaben an, sie seien im Aufklärungsgespräch über Behandlungsalternativen informiert worden, und fast alle glaubten, dass die Operation die einzige Behandlungsmöglichkeit sei. Dies dürfte mit dem Aufklärungszeitpunkt zusammenhängen. So wurde die Operationsindikation bereits vor dem Spitaleintritt gestellt und fast alle Patientinnen wurden schon durch den zuweisenden Arzt über den bevorstehenden Eingriff informiert.

Durch die Kombination von schriftlicher und mündlicher Aufklärung wurde eine hohe Patientinnenzufriedenheitsrate erzielt. Nur 2\% der Patientinnen waren unzufrieden. In der multivariaten Analyse waren türkische und serbokroatische Muttersprache mit Unzufriedenheit assoziiert. Dies entspricht amerikanischen Studien [37, 38], dass nicht englischsprechende Patienten und solche mit nur beschränkten Englischkenntnissen mit ihrer Behandlung deutlich weniger zufrieden sind. Ferner war in unserer multivariaten Analyse eine schlechte schriftliche Beschreibung der geplanten Operation ein wesentlicher Grund für Unzufriedenheit der Patientinnen mit der Operationsaufklärung.

Einige unserer Patientinnen wünschten zusätzliche Informationen hinsichtlich Diagnose (18\%), Operationstechnik (11\%) und Risiken (13\%). Dies unterstreicht die Grenzen vorgefertigter Aufklärungsprotokolle. Auf die individuellen Bedürfnisse des einzelnen Patienten kann nicht genügend eingegangen werden und das Einführen komplexerer Aufklärungsprotokolle steht im Widerspruch zum Bedürfnis nach einem persönlichen Gespräch [39]. Vor einem Eingriff sind die Patienten in einer ungewöhnlichen, belastenden Situation, was oft zu Kummer und Angst führt. Dies wird durch die Ungewissheit vor der Zukunft zusätzlich verstärkt. Zur Berücksichtigung der individuellen Bedürfnisse des Patienten ist das Gespräch zwischen Patient und Arzt unerlässlich. Nur dadurch kann es zu einem echten Dialog und einer Vertiefung der Patienten-Arzt-Beziehung kommen.

Trotz grossangelegter Multizenterstudie sind gewisse Einschränkungen zu erwähnen. Von den 6970 während des Studienzeitraumes behandelten Frauen wurden nur 3888 (56\%) in die 
Studie eingeschlossen. Eine genaue Analyse der verschiedenen Gründe, die zu dem hohen Prozentsatz an Nicht-Studien-Teilnehmerinnen führte, war wegen des Studiendesigns nicht möglich. Einerseits dürften Ärzte aus Zeitmangel ihre Patientinnen nicht in die Studie eingeschlossen und andererseits Patientinnen die Studienteilnahme abgelehnt haben. Zudem wurden vermutlich Patientinnen in Notfallsituationen, die einer sofortigen Behandlung bedurften, nicht in die Studie aufgenommen. So kann letztlich ein positiver Selektionsbias mit Überrepräsentierung zufriedener Patientinnen nicht vollständig ausgeschlossen werden. Allerdings ist zu berücksichtigen, dass eine Erhöhung der Studienteilnehmerrate von 30\% auf 70\% bei der Patienteneinschätzung einer Behandlung die Schlussfolgerungen nur minimal beeinflusst [40].

\section{Schlussfolgerung}

Die enge Zusammenarbeit von SGGG, Rechtsdienst der FMH und SPO hat sich als äusserst vorteilhaft erwiesen. Die kombinierte schriftliche und mündliche Operationsaufklärung entspricht den Wünschen und Bedürfnissen der Patientinnen. Sie ermöglicht ein strukturiertes Gespräch, erleichtert die Dokumentation und ist für Patientin und Arzt ein gültiges Rechtsdokument, dass eine angemessene Aufklärung erfolgte. So ist in der Schweiz die kombinierte schriftliche und mündliche Operationsaufklärung der SGGG als Goldstandard anzusehen.

\section{Danksagung}

Die Schweizerische Patientenorganisation dankt der Schweizerischen Gesellschaft für Gynäkologie und Geburtshilfe und den mitwirkenden 11 Kliniken für Gynäkologie und Geburtshilfe sowie der Gesundheitsförderung Schweiz für die Durchführung dieser Studie. Ein besonderer Dank gebührt Prof. Dr. med. Urs Haller, ehemaliger Vorsteher des Departements für Frauenheilkunde des Universitätsspitals Zürich, der die Studie tatkräftig unterstützte.

\section{Literatur}

1 Ghulam AT, Kessler M, Bachmann LM, Haller U, Kessler TM. Patients' satisfaction with the preoperative informed consent procedure: a multicenter questionnaire survey in Switzerland. Mayo Clin Proc. 2006;81:307-12.

2 Hopper KD, Ten Have TR, Hartzel J. Informed consent forms for clinical and research imaging procedures: how much do patients understand? AJR. 1995;164:493-6.
3 Hopper KD, Ten Have TR, Tully DA, Hall TE. The readability of currently used surgical/procedure consent forms in the United States. Surgery. 1998; 123:496-503.

4 McCormack D, Evoy D, Mulcahy D, Walsh M. An evaluation of patients comprehension of orthopaedic terminology: implications for informed consent. J R Coll Surg Edinb. 1997;42:33-5.

5 Lynöe N, Sandlund M, Dahlqvist G, Jacobsson L. Informed consent: study of quality of information given to participants in a clinical trial. BMJ. 1991; 303:610-3.

6 Lloyd AJ, Hayes PD, London NJM, Bell PRF, Naylor AR. Patients' ability to recall risk associated with treatment options. Lancet. 1999;353:645.

7 Gattellari M, Butow PN, Tattersall MH. Informed consent: what did the doctor say? Lancet. 1999; 353:1713.

8 Guillod O. Pour une meilleure information des patients: des brochures écrites. Schweiz Ärztezeitung. 1995;76:318-21.

9 Meuli H, Kuhn H-P. Aufklärungspflicht: Weder Verängstigung noch Verdrängung. Schweiz Ärztezeitung. 1995;76:311.

10 Payllier P. Rechtsprobleme der ärztlichen Aufklärung. Unter besonderer Berücksichtigung der spitalärztlichen Aufklärung. Vol. 145. Zürcher Studien zum Privatrecht, Zürich: Schulthess Polygraphischer Verlag Zürich, 1999:83ff

11 Haller U, Hepp H, Reinold E, Winter R. How much preoperative information? - How can it be documented? Gynakol Geburtshilfliche Rundsch. 1999;39:181-2.

12 Kessler W, Faisst K, Kessler M, Aeberhard P, Ammann J, Biaggi J, Decurtins M, Schweizer W. Quality control in patient education. Results of a patient survey about the patient education protocol of the Swiss Society of Surgery in 6 Swiss hospitals. Swiss Surg. 2000;6:42-9; quiz 50-3.

13 Flory J, Emanuel E. Interventions to improve research participants' understanding in informed consent for research: a systematic review. JAMA. 2004;292:1593-601.

14 Bertschinger P. Informed consent. Schweiz Ärztezeitung. 2002;83:603-5.

15 Hoermann S, Doering S, Richter R, Walter $\mathrm{MH}$ Schussler G. Patients' need for information before surgery. Psychother Psychosom Med Psychol 2001;51:56-61.

16 Kessler TM, Nachbur BH, Kessler W. Patients' perception of preoperative information by interactive computer program-exemplified by cholecystectomy. Patient Educ Couns. 2005; 59:135-40.

17 Bates T. Ethics of consent to surgical treatment. Br J Surg. 2001;88:1283-4.

18 Shepherd HA, Bowman D, Hancock B, Anglin J, Hewett D. Postal consent for upper gastrointestinal endoscopy. Gut. 2000;46:37-9.

19 Askew G, Pearson KW, Cryer D. Informed consent: can we educate patients? J R Coll Surg Edinb. 1990;35:308-10. 
20 Lewis PJ, O'Keefe L, Adcock S. Patients who were given information sheets have better postoperative recall of information. J R Coll Surg Edinb. 1991; $36: 206-7$

21 Edwards MH. Satisfying patients' needs for surgical information. Br J Surg. 1990;77:463-5.

22 Eden OB. Consenting patients. BMJ. 1990;301: 1334

23 Cassell EJ, Leon AC, Kaufman SG. Preliminary evidence of impaired thinking in sick patients. Ann Intern Med. 2001;134:1120-3.

24 Armstrong AP, Cole AA, Page RE. Informed consent: are we doing enough? Br J Plast Surg. 1997;50:637-40.

25 Bunker TD. An information leaflet for surgical patients. Ann R Coll Surg Engl. 1983;65:242-3.

26 Stenchever Morton A. Too much informed consent? Obstet Gynecol. 1991;77:631.

27 Schindele S, Renggli P, Hackenbruch W. Patient education before orthopedic elective interventions. "The Langenthal model of 3-step patient education». Swiss Surg. 2001;7:151-7.

28 Dawes PJ, Davison P. Informed consent: what do patients want to know? J R Soc Med. 1994;87: 149-52.

29 Aabakken L, Baasland I, Lygren I, Osnes M. Development and evaluation of written patient information for endoscopic procedures Endoscopy. 1997;29:23-6.

30 Dawes PJ, O'Keefe L, Adcock S. Informed consent: the assessment of two structured interview approaches compared to the current approach. J Laryngol Otol. 1992;106:420-4.
31 Kerrigan DD, Thevasagayam RS, Woods TO, McWelch I, Thomas WE, Shorthouse AJ, Dennison AR. Who's afraid of informed consent? BMJ. 1993; 306:298-300.

32 Beddows J. Alleviating pre-operative anxiety in patients: a study. Nurs Stand. 1997;11:35-8.

33 Sanwal AK, Kumar S, Sahni P, Nundy S. Informed consent in Indian patients. J R Soc Med. 1996; 89:196-8.

34 Coulter A. Patients' views of the good doctor BMJ. 2002;325:668-9.

35 Pimentel FL, Ferreira JS, Vila Real M, Mesquita NF Maia-Goncalves JP. Quantity and quality of information desired by Portuguese cancer patients. Support Care Cancer. 1999;7:407-12.

36 Mulcahy D, Cunningham K, McCormack D, Cassidy N, Walsh M. Informed consent from who? J R Coll Surg Edinb. 1997;42:161-4.

37 Carrasquillo O, Orav EJ, Brennan TA, Burstin HR. Impact of language barriers on patient satisfaction in an emergency department. J Gen Intern Med. 1999;14:82-7.

38 Weech-Maldonado R, Morales LS, Elliott M, Spritzer K, Marshall G, Hays RD. Race/ethnicity, language, and patients' assessments of care in Medicaid managed care. Health Serv Res. 2003 38:789-808.

39 Akkad A, Jackson C, Kenyon S, Dixon-Woods M, Taub N, Habiba M. Informed consent for elective and emergency surgery: questionnaire study. BJOG. 2004;111:1133-8.

40 Perneger TV, Chamot E, Bovier PA. Nonresponse bias in a survey of patient perceptions of hospital care. Med Care. 2005;43:374-380. 